\title{
Highly unquenched orbital moment in textured Fe-phthalocyanine thin films
}

\author{
J. Bartolomé, ${ }^{1, *}$ F. Bartolomé, ${ }^{1}$ L. M. García, ${ }^{1}$ G. Filoti, ${ }^{2}$ T. Gredig, ${ }^{3}$ C. N. Colesniuc, ${ }^{4}$ I. K. Schuller, ${ }^{4}$ and J. C. Cezar ${ }^{5}$ \\ ${ }^{1}$ Instituto de Ciencia de Materiales de Aragón, and Departamento de Física de la Materia Condensada, \\ CSIC-Universidad de Zaragoza, 50009 Zaragoza, Spain \\ ${ }^{2}$ National Institute for Materials Physics, P.O. Box MG 7, 077125 Bucharest-Magurele, Romania \\ ${ }^{3}$ Department of Physics and Astronomy, California State University, Long Beach, California 90840, USA \\ ${ }^{4}$ Department of Physics, University of California at San Diego, California 92093, USA \\ ${ }^{5}$ European Synchrotron Radiation Facility, BP 220, F-38043 Grenoble, France
}

(Received 28 October 2009; revised manuscript received 29 March 2010; published 5 May 2010)

\begin{abstract}
X-ray magnetic circular dichroism (XMCD) measured at $T=6 \mathrm{~K}$ and $\mu_{0} H=5 \mathrm{~T}$ on the $\alpha$-phase Fephthalocyanine $(\mathrm{FePc})$ textured thin films shows that the $\mathrm{Fe}^{2+}$ ions present an unusually large, highly unquenched $m_{L}=0.53 \pm 0.04 \mu_{B}$ orbital component, with planar anisotropy. The spin $m_{S}=0.64 \pm 0.05 \mu_{B}$ and the intra-atomic magnetic dipolar $m_{T}$ components were also obtained. The $m_{L} / m_{S}=0.83$ ratio is the largest measured in $3 d$ complexes and compounds. The origin of this unusually high orbital moment is the incompletely filled $e_{g}$ level lying close to the Fermi energy. This explains the unusually large and positive hyperfine field detected by Mössbauer spectroscopy in FePc. The FePc film strong planar anisotropy inferred from XMCD experiments is fully confirmed by magnetization measurements.
\end{abstract}

DOI: 10.1103/PhysRevB.81.195405 PACS number(s): 75.25.-j, 68.55.am, 78.70.Dm, 81.15.Hi

\section{INTRODUCTION}

Magnetic coordination compounds are of high interest because they show peculiar quantum properties, that can be chemically tuned to modify their properties to be used for high density data storage. A detailed understanding of their specific magnetic interactions may suggest, for example, routes for processing and tailoring room temperature molecular ferromagnets. However, probing particular atoms within a magnetic molecule is often difficult and, therefore, information about interactions in covalently or coordination bonded magnetic molecules is largely lacking. Metal Phthalocyanines are simple model molecules for the understanding of the electronic properties of metals in complexes. ${ }^{1}$ In spite of extensive work on them their magnetic properties are not fully understood. Therefore, unambiguous experimental determinations are the primary tools to challenge various theoretical predictions. In particular, the orbital moment is the main physical contribution to magnetic anisotropy. Consequently, discerning the mechanisms that control the expectation value of the orbital moment $\left\langle L_{z}\right\rangle$ is of paramount importance in mastering the properties of magnetic systems for basic and applied magnetism. X-ray magnetic circular dichroism (XMCD) is the tool of choice for such a study since it is element selective and capable of resolving the orbital and spin component of the magnetic moment.

Since the early days of quantum mechanics it was found that in solids of local low-symmetry environments the angular momentum is quenched by crystal field (CF) effects. ${ }^{2}$ This was proven more recently by XMCD in $\mathrm{Fe}_{8}$ (Ref. 3) and $\mathrm{Mn}_{12}$-acetate ${ }^{3,4}$ molecular magnets, in which the orbital moment is negligible as a result of the $\left| \pm L_{z}\right\rangle$ mixing induced by the $\mathrm{CF}$ interaction acting on the magnetic atom. On the other hand, the spin-orbit coupling splits the CF spin degenerate levels and a nonzero orbital moment contribution appears in the magnetic ground state. Usually the orbital moment is small compared to the spin component, but a remarkable enhancement of the orbital moment of magnetic systems was observed at film interfaces, ${ }^{5}$ monoatomic metal chains, ${ }^{6}$ nanoparticles, ${ }^{7}$ or very small clusters. ${ }^{8}$ In the limit of atomic size, that is, as atomic impurities in solids, the orbital moment may be only partially quenched, with values approaching the free atom ones. ${ }^{9,10}$

In this paper, in drastic contrast to all above cases, we present a direct proof of the presence of a very large, highly unquenched orbital moment $m_{L}=0.53 \pm 0.04 \mu_{B}$ in a lowsymmetry, planar Fe(II)-Phthalocyanine (FePc) molecule.

In $\mathrm{FePc}$, the $\mathrm{Fe}$ atom has square-planar coordination with four pyrrolic $N$ atoms. In the bulk and thin films these planar molecules form linear chains by columnar stacking. The molecule fourfold axis is tilted with respect to the crystal $b$ axis by an angle $\phi$. In the bulk form only two phases appear; the $\alpha$-FePc with a tilting angle of $\phi=26.5^{\circ}$, and the $\beta$ phase with $\phi=44.8^{\circ}$. The former becomes ferromagnetic below $T_{C} \sim 10 \mathrm{~K}$ in the bulk form while the latter is paramagnetic down to liquid helium temperatures. The magnetic properties were satisfactorily interpreted in terms of an effective spin $S=1 \mathrm{Fe}(\mathrm{II})$ model. ${ }^{11}$

\section{EXPERIMENTAL DETAILS}

Thin films of FePc are deposited on a smooth gold substrate with the molecule plane lying flat on the substrate plane. ${ }^{12}$ The thin film sample in this work was prepared in an organic molecular beam epitaxy (OMBE) system. SigmaAldrich FePc was purified in three cycles using the temperature gradient method. The purified material was loaded into a vacuum chamber and out-gassed for several hours before deposition. The chamber pressure during the deposition was $1.2 \times 10^{-8}$ Torr with the base pressure around 5 $\times 10^{-9}$ Torr.

In thin films grown by OMBE the molecule plane orientation is set by a proper substrate selection and the growth conditions. ${ }^{13,14}$ In order to obtain $\alpha$-phase FePc films with 
the molecule plane parallel to the sapphire substrate, first a $40 \mathrm{~nm}$ nominal thickness Au buffer layer was deposited at a rate of $0.3 \AA / \mathrm{s}$. This buffer layer was annealed for one hour at $300{ }^{\circ} \mathrm{C}$ "in situ" to remove any water from the surface and to smoothen the Au surface. Without breaking vacuum, the FePc was deposited from a crucible set to $350{ }^{\circ} \mathrm{C}$, while the substrate was maintained at $150{ }^{\circ} \mathrm{C}$ to increase the crystallite size. The typical growth rate was $0.9 \AA$ /s. The FePc deposition rate was determined by means of a quartz crystal monitor before and after the deposition. The nominal thickness was then calculated by comparison to a thickness calibrated FePc sample. The FePc thickness of the investigated sample was $80 \mathrm{~nm}$.

The $\mathrm{x}$-ray linear polarized absorption (XLPA) and XMCD experiments were performed at the ID08 beamline of the European Synchrotron Radiation Facility (ESRF) in Grenoble. A spherical grating monochromator was used. The photon flux at the sample was $10^{12}$ photons per second in a $5 \times 10^{-4}$ bandwidth. The energy resolution at the Fe- $L_{2,3}$ energy region is about $\Delta E / E=5 \times 10^{-4}$. The detection technique was total electron yield. In both experiments a monochromatic x-ray beam impinges the sample with varying incidence angles $\gamma$ with the normal to the substrate and the molecule plane (see inset in Fig. 2) within the range $0^{\circ}$ $<\gamma<75^{\circ}$. In XLPA, the light is linearly polarized while in $\mathrm{XMCD}$ it is right- and left-circularly polarized. The XMCD experiment was performed with $\mu_{0} H=1,3$, and $5 \mathrm{~T}$ applied magnetic field. To avoid experimental artifacts, the four combinations of field direction and circular polarization are recorded for each $\gamma$ angle. The XMCD signal is the difference between absorption spectra recorded with the helicity antiparallel and parallel to the field. In all cases, the polarization rate was well above $99 \%$. All experiments were carried out at $T=6 \mathrm{~K}$. No radiation damage of the sample was detected.

Magnetization measurements have been performed in a superconducting quantum interference device (SQUID) magnetometer, with the high sensitivity option, on a platelet of $2 \times 2 \mathrm{~mm}^{2}$, in the direction parallel $\left(M_{x y}\right)$ and perpendicular $\left(M_{z}\right)$ to the substrate plane, in the range 1.8 to $10 \mathrm{~K}$.

\section{FILM MORPHOLOGY}

The $\theta-2 \theta$ diffraction patterns of the FePc film deposited onto gold plated sapphire (Fig. 1) shows a pronounced (313) peak at $2 \theta=27.84^{\circ}$ indicating that the $b$ axis of the molecule is perpendicular to the substrate plane. Note that the (200) Bragg peak at $2 \theta=6.8^{\circ}$, observed in a bulk powder pattern, is absent in the film where the molecule is deposited onto a gold surface. These two features show that the molecule planes are parallel to the substrate due to interaction between $\mathrm{Au}$ and the first FePc layer. This layered configuration is shown in the inset of Fig. 2. Atomic force microscopy and $\mathrm{X}$-ray diffraction proved that the samples consist of textured columnar grains with a random distribution of the molecular $x$ and $y$ axes parallel to the substrate plane and a small mosaic spread in the $z$ direction. ${ }^{13}$

In the XLPA experiments the polarization of $\vec{E}$ is denoted as horizontal $(H)$ and vertical $(V)$ for parallel and perpendicular to the storage ring electron orbit plane, respectively.

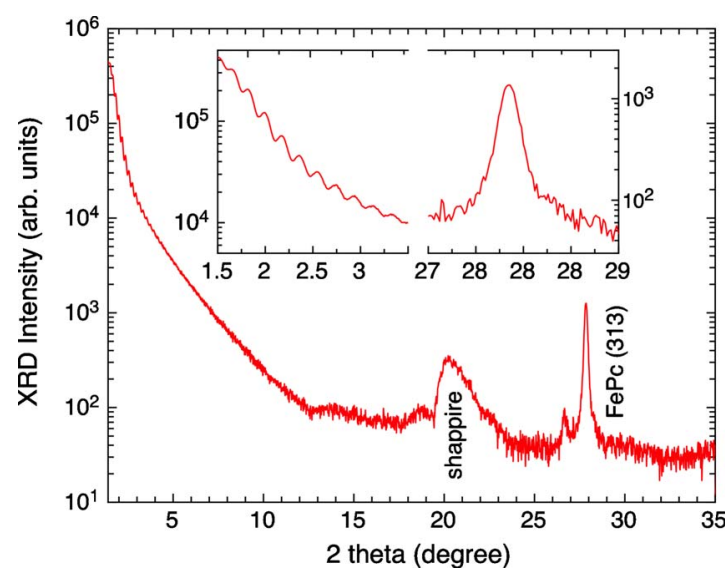

FIG. 1. (Color online) $\theta-2 \theta$ diffraction patterns of FePc film grown on a gold surface. The oscillations at low diffraction angles correspond to finite-size oscillations from the thin film. The inset shows an expanded view of the oscillations and the FePc (313) peak. The small peak at $26.6^{\circ}$ is the same FePc (313) peak due to a tungsten impurity on the copper $\mathrm{x}$-ray source.

Besides the X-ray diffraction measurement cited above, the XLPA measurements performed at the $N$ K-edge at normal and grazing $\left(75^{\circ}\right.$ from normal) incidence (shown in Fig. 2) confirm that the molecules lay parallel to the substrate plane. Indeed, the spectra in normal incidence with $\vec{E}_{H}$ or $\vec{E}_{V}$ polarization are identical, and also equal to the spectra with $\vec{E}_{V}$ polarization in grazing incidence, as expected for $\vec{E}$ lying in the molecule plane. On the other hand, the spectra with $\vec{E}_{H}$ in grazing incidence has $\vec{E}$ oriented $75^{\circ}$ from the molecule plane, and the resulting spectra $H$ and $V$ in grazing incidence are very different, as shown in Fig. 2. Thus it is possible the

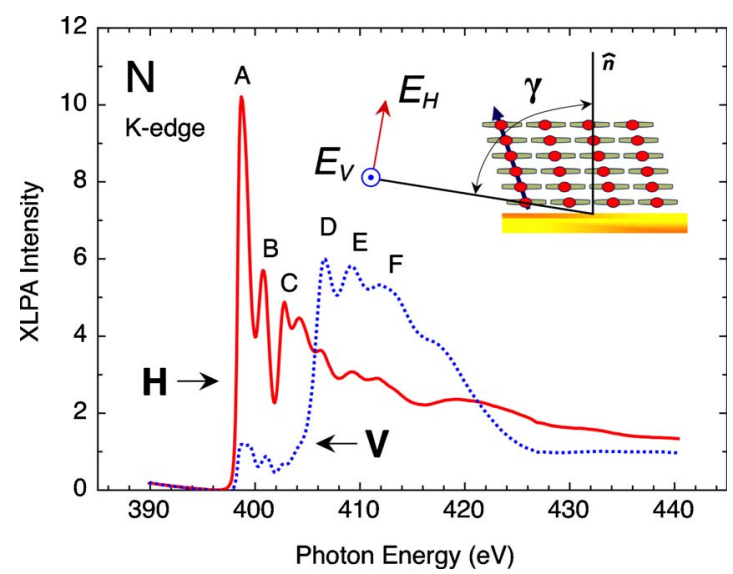

FIG. 2. (Color online) XLPA spectra recorded at the $N$ K-edge with grazing angle incidence $\left(\gamma=75^{\circ}\right)$ at $T=6 \mathrm{~K}$. The two spectra correspond to the $H$ and $V$ polarization modes: $\vec{E}$ is either parallel to the FePc plane ( $V$, dashed line) or forming an angle $15^{\circ}$ with the normal to the substrate, i.e., to the $\mathrm{FePc}$ plane $(H$, full line). In accordance with Ref. 15 , peaks $A, B$, and $C$ are assigned to $\pi^{*}$ resonances, and $D, E$, and $F$ to $\sigma^{*}$ ones. Inset: schematic view of sample FePc deposited on a Au film, previously deposited on sapphire. The incoming beam at grazing incidence is shown with the $\vec{E}$ in the $H$ and $V$ polarization modes. 


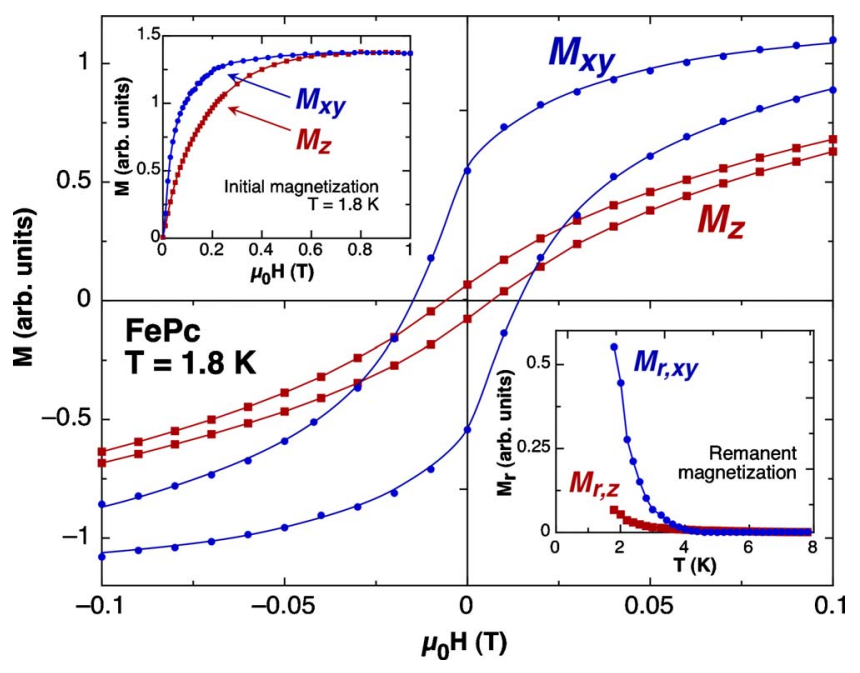

FIG. 3. (Color online) Hysteresis loop of the FePc film deposited on $\mathrm{Au}$ covered sapphire measured at $T=1.8 \mathrm{~K}$ in the direction perpendicular $\left(M_{z}\right)$ and parallel $\left(M_{x y}\right)$ to the substrate. Insets: (a) upper left; initial magnetization at $T=1.8 \mathrm{~K}$, in the two directions; (b) lower right; remanent moment $M_{r}$ as a function of temperature.

assignment of the peaks (see caption of Fig. 2) in good agreement with that proposed for NiPc deposited on $\mathrm{Cu}(110) .{ }^{15}$ The $\pi^{*}$ resonances are found at $398.7,400.3$, and $402.3 \mathrm{eV}$ and the $\sigma^{*}$ resonances at 406.2, 409.3, and 411.6 $\mathrm{eV}$. The near disappearance of the $\pi^{*}$ resonances when $\vec{E}$ is in the molecule plane demonstrates that the four $N$ atoms lay in a plane parallel to the substrate.

\section{MAGNETIC CHARACTERIZATION}

Initial magnetization, hysteresis cycles up to $H=5 \mathrm{~T}$ and thermoremanence have been measured from $T=1.8 \mathrm{~K}$ to 10 $\mathrm{K}$, parallel and perpendicular to the FePc film. In Fig. 3 we show $M_{x y}$ and $M_{z}$ in the low-field regime at $T=1.8 \mathrm{~K}$. The open $M_{x y}$ hysteresis cycle demonstrates that the film is ferromagnetic at this temperature. The coercive field is $\mu_{0} H_{c}$ $=0.145 \mathrm{mT}$. Besides, $M_{x y}$ is higher than $M_{z}$ up to $0.6 \mathrm{~T}$, coalescing at this field. This feature is indicative of planar $(x y)$ anisotropy; i.e., the easy axis of magnetization is parallel to the substrate. The initial magnetization curves; i.e., when the sample was demagnetized before the $M(H)$ experiment was done, is shown in inset (a). The $M_{x y}$ curve has a higher slope at $H=0$ than the $M_{z}$ one, again, indicating $x y$ anisotropy. The anisotropy field can be roughly estimated, from the intercept of the $M_{x y}$ and $M_{z}$ curves, therefore, the anisotropy field is $\mu_{0} H_{A}=0.6 \mathrm{~T}$. The remanence magnetization dependence on temperature $M_{r}(T)$ is included in inset (b) of Fig. 3. $M_{r}$ remains nonzero; i.e., ferromagnetic, up to $T_{C} \approx 4 \mathrm{~K}$, in the $x y$ plane. Note that, the Curie temperature of this FePc film is lower than the reported $T_{C}=10 \mathrm{~K}$ for the bulk $\alpha$-FePc. ${ }^{11}$ This decrease in $T_{C}$ could be expected as a size effect due to the reduced $(80 \mathrm{~nm})$ thickness of the film. The hysteresis curves at $6 \mathrm{~K}$ have no open loops (not shown), but still $M_{x y}>M_{z}$ up to $0.6 \mathrm{~T}$, so the $x y$ anisotropy remains in the paramagnetic region near $T_{C}$. Our XMCD measurements performed at $T=6 \mathrm{~K}$, were, therefore, per-

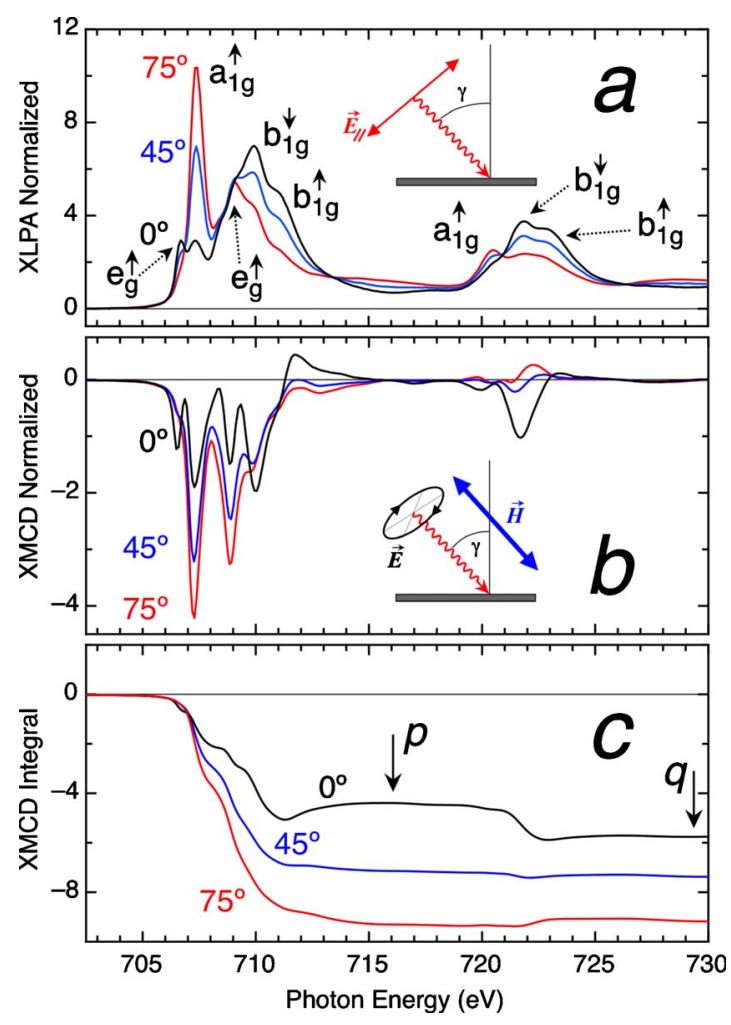

FIG. 4. (Color online) (a) normalized XLPA, (b) XMCD spectra, and (c) integrated XMCD area at the $\mathrm{Fe} L_{2,3}$ edges on $\mathrm{FePc}$ at $T=6 \mathrm{~K}$ for incidence angle $\gamma=0^{\circ}, 45^{\circ}$, and $75^{\circ}$. In the XMCD experiment, the applied field $\left(\mu_{0} H=5 \mathrm{~T}\right)$ and the helicity of the beam are parallel for every $\gamma$. The arrows indicate the higher limit of the $p$ and $q$ integrals.

formed in the paramagnetic phase of the material. The main conclusion of this section is that the FePc film has magnetic planar anisotropy at low temperatures.

\section{X-RAY SPECTROSCOPY RESULTS}

Panels $a$ and $b$ of Fig. 4 display, respectively, selected normalized XLPA and XMCD spectra measured at the $L_{2,3}$ Fe edges. In XLPA (panel $a$ of Fig. 4) the absorption intensity is proportional to the number of empty valence states in the direction of the electric field $\vec{E},{ }^{16}$ which is parallel to the molecule plane for $\gamma=0^{\circ}$ and nearly perpendicular to it for grazing incidence $\left(\gamma \approx 90^{\circ}\right)$. Since there is a random distribution of molecules in the $x y$ plane, the spectra are averaged over all azimuthal angles, while all molecules have a (nearly) common $z$. XMCD (panel $b$ of Fig. 4) provides information on the polarized magnetic moment per $\mathrm{Fe}$ atom of the initial state.

The electronic ground state has been proposed to be ${ }^{3} E_{g},{ }^{17-20}$ among others. In the following, we show that our experimental results do support this assignment.

Indeed, taking into account the corresponding ${ }^{3} E_{g}$ molecular orbital level scheme (shown in Fig. 5), the $e_{g}^{\uparrow}$ level splitting explained in Ref. 19 (see below), and the sign of the XMCD signals, several features of the XLPA spectra can be assigned. 


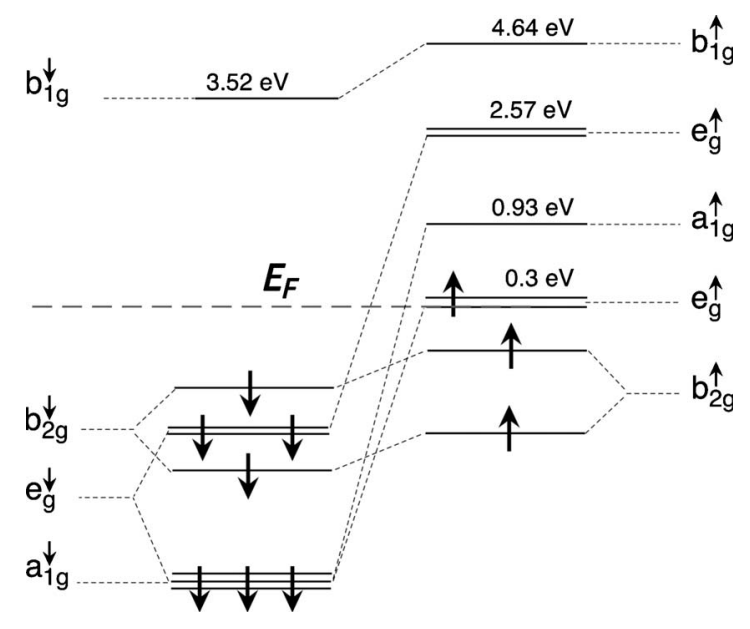

FIG. 5. Spin split molecular orbital energy level scheme for FePc (Ref. 17). For clarity, only the states with a relevant component of $3 d$ weight $\left(w_{3 d}>0.05\right)$ have been included. Note also the global change in spin sign with respect to Ref. 17.

The Fermi energy $\left(E_{F}\right)$ is taken as the energy of the maximum slope of the upturn at the $L_{3}$ edge. The $L_{3}$ spectrum $\left(2 p_{3 / 2} \rightarrow 3 d\right.$ transitions) shows a first peak at $706.4 \mathrm{eV}$, which may be ascribed to the $e_{g}^{\uparrow}$ empty level, with mixing of the $a_{1 g}^{\uparrow}$ level. This sets the lowest unoccupied molecular orbital (LUMO) at energy $0.3 \mathrm{eV}$ above $E_{F}$. The next most intense peak at $707.3 \mathrm{eV}$ with $\vec{E}$ close to $z\left(\gamma=75^{\circ}\right)$, nearly disappears when $\vec{E} \| x, y\left(\gamma=0^{\circ}\right)$, and therefore it may be identified with a transition to the $d_{3 z^{2}-r^{2}}$, i.e., the empty antibonding excited level $a_{1 g}^{\uparrow}$ (see Fig. 5) lying $0.93 \mathrm{eV}$ above $E_{F}$.

On the other hand, at the high energy side of the $L_{3}$ spectra a triplet of peaks can be identified. The leftmost peak at $708.9 \mathrm{eV}$ does not depend on $\gamma$, and is assigned to an $e_{g}^{\uparrow}$ state in the minority antibonding states stemming from hybridization with the $\pi$ ligand orbitals $(2.57 \mathrm{eV})$ : The two on the right side (709.8 and $711.7 \mathrm{eV})$ reduce their intensity when $\vec{E} \| z$ and may be indexed as due to the $d_{x^{2}-y^{2}}$ states at the empty majority $b_{1 g}^{\downarrow}(3.52 \mathrm{eV})$ and minority $b_{1 g}^{\uparrow}$ antibonding states $(4.64 \mathrm{eV})$, respectively. The $L_{2}$ edge spectra $\left(2 p_{1 / 2}-3 d\right.$ transitions) are less resolved. However, by comparison with the $L_{3}$ edge spectra, considering their relative energies and angle dependences, the left one can be assigned to the $a_{1 g}^{\uparrow}$ excited state and the two higher energy ones to the $\left(d_{x^{2}-y^{2}}\right) b_{1 g}^{\downarrow}$ and $b_{1 g}^{\uparrow}$ antibonding states. A more detailed description of the XLPA spectra requires a multiplet calculation, which is beyond the scope of this paper.

Information on the orbital and spin components of the magnetic moment of the absorbing atom projected along the field direction for a given incidence angle $\gamma$ can be obtained from the sum rules ${ }^{21,22}$

$$
\begin{gathered}
\frac{m_{L}(\gamma)}{\mu_{B}}=-\frac{2 n_{h}}{r} \int\left(\Delta \mu_{L_{3}}+\Delta \mu_{L_{2}}\right) d E, \\
\frac{m_{S}^{\text {eff }}(\gamma)}{\mu_{B}}=-\frac{3 n_{h}}{r} \int\left(\Delta \mu_{L_{3}}-2 \Delta \mu_{L_{2}}\right) d E,
\end{gathered}
$$

where the XMCD at the $L_{2,3}$ edges is $\Delta \mu_{L_{2,3}}=\mu_{L_{2,3}}^{-}-\mu_{L_{2,3}}^{+}$, being $\mu_{L_{2,3}}^{-}$and $\mu_{L_{2,3}}^{+}$the absorption measured with left $(-)$ and right $(+)$ circularly polarized light. $n_{h}$ is the number of $\mathrm{d}$ holes above the Fermi energy, which has been calculated for $\mathrm{FePc}$ as $n_{h}=2.669 .{ }^{17}$

In the following paragraphs the data analysis for $T=6 \mathrm{~K}$ and $\mu_{0} H=5 \mathrm{~T}$ is shown in detail. The analysis for $\mu_{0} H=1$ and $3 \mathrm{~T}$ are analyzed likewise. After removal of the contributions from transitions to the continuum by subtraction of two arctangent functions centered at the $L_{3}$ and $L_{2}$ edges and $2 / 3$ and $1 / 3$ amplitude, respectively, $r$ is calculated as $r$ $=\int\left(\mu^{+}+\mu^{-}+\mu^{0}\right) d E$, the integrated spectrum for the unpolarized radiation. $\frac{1}{2} \int\left(\mu^{+}+\mu^{-}\right) d E=I_{L 2}+I_{L 3}$ is the integrated unpolarized x-ray absorption white-line intensity. $\mu^{0}$ has been approximated by $\frac{1}{2}\left(\mu^{+}+\mu^{-}\right)$.

In Fig. 4(c) the values of the integrated XMCD, $p$ $=\int\left(\Delta \mu_{L_{3}}\right) d E$ and $q=\int\left(\Delta \mu_{L_{3}}+\Delta \mu_{L_{2}}\right) d E$, with upper integration limits at the respective arrows, have been depicted. The orbital sum rule [Eq. (1)] directly yields the orbital moment component, $m_{L}(\gamma)=-\left\langle L_{z}\right\rangle(\gamma) \mu_{\mathrm{B}} / \hbar$. From the spin sum rule [Eq. (2)] and the relation $m_{S}^{\text {eff }}(\gamma)=m_{S}-7 m_{T}(\gamma)$ one obtains the isotropic spin moment, $m_{S}=-2\langle S\rangle \mu_{\mathrm{B}} / \hbar$, and the intraatomic magnetic dipolar component, $m_{T}(\gamma)=\left\langle T_{\gamma}\right\rangle \mu_{B} / \hbar$.

The measured XMCD magnitude with $H$ parallel to the beam direction and incidence angle $\gamma$ furnishes the projection of the $\mathrm{Fe}$ magnetic moment along the direction of the applied field, $m(\gamma)=\vec{H} \cdot \vec{m} / H$. The FePc molecule symmetry may be approximated to $D_{4 h}$, therefore the fourfold axis may be taken as the principal axis, $z$, with the two binary axes $x$ and $y$ assumed identical.

In the case of a molecule lying flat on the substrate, and the $x$ axis at an angle $\alpha$ with respect to a fixed reference axis parallel to the substrate, the measured magnetic moment can be decomposed into two terms:

$$
m(\gamma)=m^{z} \cos ^{2} \gamma+\left(m^{x} \sin ^{2} \alpha+m^{y} \cos ^{2} \alpha\right) \sin ^{2} \gamma .
$$

Due to the random distribution of the molecular axes parallel the substrate, averaging $\alpha$ between 0 and $2 \pi$ one obtains

$$
m(\gamma)=m^{z} \cos ^{2} \gamma+m^{x y} \sin ^{2} \gamma,
$$

where we have defined $m^{x y}=(1 / 2)\left(m^{x}+m^{y}\right)$, the mean of the magnetization of the molecule in the $x$ and $y$ axes.

The magnetic moment $m$ is decomposed into its orbital component

$$
m_{L}(\gamma)=m_{L}^{z} \cos ^{2} \gamma+m_{L}^{x y} \sin ^{2} \gamma
$$

and the effective spin component

$$
m_{S}^{\text {eff }}(\gamma)=m_{S}-7\left(m_{T}^{z} \cos ^{2} \gamma+m_{T}^{x y} \sin ^{2} \gamma\right) .
$$

In $D_{4 h}$ symmetry the diagonal tensor components fulfill the relation $\sum_{i=x, y, z} T_{i i}=0$, therefore $\left(m_{T}^{z}+2 m_{T}^{x y}\right)=0$ and only four moment components in Eqs. (5) and (6) are independent. In particular, we measured XMCD at the so-called magic angle $\gamma^{*}=54.7^{\circ}$ (Ref. 23) for which $2 \cos ^{2} \gamma^{*}$ $=\sin ^{2} \gamma^{*}$. At the incidence angle $\gamma^{*}$ the intra-atomic dipolar contribution cancels for the $D_{4 h}$ symmetry, giving a direct measure of $m_{S} \cdot{ }^{23}$

In Fig. 6 the $m_{L}$ and $m_{S}^{\text {eff }}$ obtained at $T=6 \mathrm{~K}$ and $\mu_{0} H$ $=5 \mathrm{~T}$ from the sum rules as a function of $\gamma$ are shown. The excellent fitting of the experimental results to Eqs. (5) and 


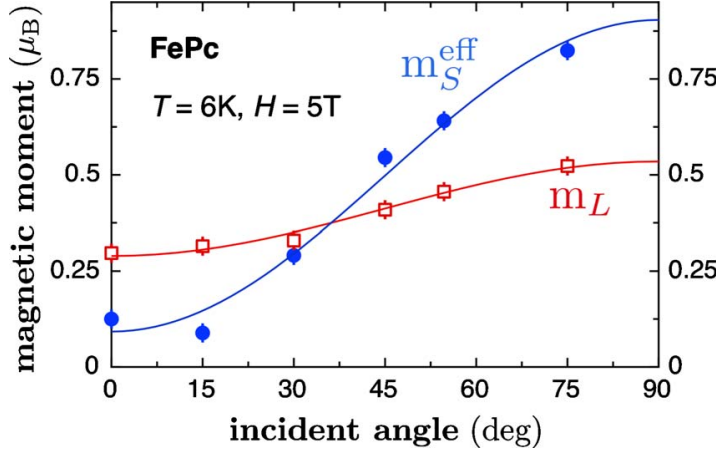

FIG. 6. (Color online) $m_{L}(\square)$ and $m_{S}^{\text {eff }}(\bullet)$, as determined from the XMCD spectra at $T=6 \mathrm{~K}$ and $\mu_{0} H=5 \mathrm{~T}$. The lines show the fits to Eqs. (5) and (6).

(6) is also presented in Fig. 6. The four adjustable parameters are collected in Table I. In Fig. 7 the magnetic moments $m_{L}^{x y}$, $m_{S}$, and $m^{x y}$ for $\mu_{0} H=1,3$, and $5 \mathrm{~T}$ are depicted together with the SQUID magnetization measurements at $T=6 \mathrm{~K}$, scaled to match the XMCD data. It is evidenced that at $\mu_{0} H=5 \mathrm{~T}$ the magnetization is near saturation.

The value of the isotropic spin component $m_{S}$ $=0.64 \pm 0.05 \mu_{B}$ resulted from the fit agrees exactly with the direct determination of $m_{S}$ from the application of the effective spin sum rule at the $\gamma^{*}$ magic incidence angle. The orbital moment is anisotropic, being largest when the applied field is parallel to the $x y$ molecule plane, confirming our magnetization results. Its value is $m_{L}^{x y}=0.53 \pm 0.04 \mu_{B}$. In spite of the applied approximations, and the inherent experimental uncertainty, the existence of an extraordinarily large, highly unquenched orbital moment in FePc is proven beyond doubt. This fact also explains the Mössbauer spectroscopy (MS) as a function of field measurements, which found the highest hyperfine field of positive sign ever measured on an $\mathrm{Fe}(\mathrm{II})$ ion $\left(B_{h f}=66.2 \mathrm{~T}\right) .{ }^{19}$ Indeed, this requires the existence of a large orbital moment to explain the field dependence of the low temperature MS sextets, in excellent agreement with our result.

The ratio $m_{L}^{x y} / m_{S}=0.83$ is the largest reported on $3 d$ ions in compounds or molecular systems. The large value of the orbital moment $m_{L}^{x y}=0.53 \mu_{B}$ is comparable to that found for individual molecules of $\mathrm{O}_{2}-\mathrm{Fe}(\mathrm{TPA})_{4}$ deposited on a $\mathrm{Cu}$ surface, ${ }^{24}$ where also $x y$ anisotropy was found. However, in the $\mathrm{O}_{2}-\mathrm{Fe}(\mathrm{TPA})_{4}$ case it is argued that the interactions with the surface play an important role in the magnetic properties, in contrast with our FePc thin layer, where that interaction is negligible in comparison with the intermolecular interaction. Besides, such a large orbital moment $\left(m_{L}^{x y}=0.53 \mu_{B}\right)$ has also been found in $\mathrm{V}_{2} \mathrm{O}_{3},{ }^{25}$ but in this case the ratio $m_{L} / m_{S}$

TABLE I. The four adjusted magnetic moment parameters (in units of $\mu_{B}$ ), obtained from the fit of the XMCD sum rule results (see Fig. 6) to Eqs. (5) and (6), and the calculated orbital to spin moment ratio.

\begin{tabular}{ccccc}
\hline \hline$m_{L}^{z}$ & $m_{L}^{x y}$ & $m_{S}$ & $m_{T}^{z}$ & $m_{L}^{x y} / m_{S}$ \\
\hline $0.29(5)$ & $0.53(4)$ & $0.64(5)$ & $0.074(5)$ & $0.83(4)$ \\
\hline \hline
\end{tabular}

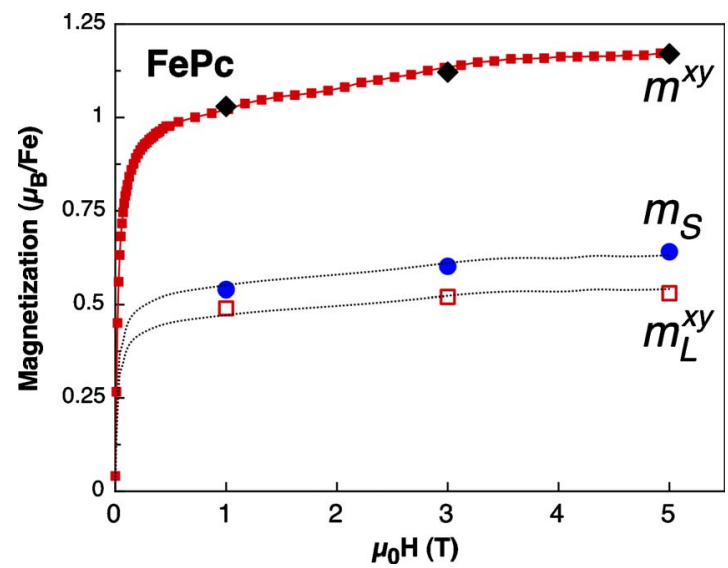

FIG. 7. (Color online) $m^{x y}(\diamond), m_{L}^{x y}(\square)$, and $m_{S}(\bullet)$ as a function of field of the FePc film, as determined from XMCD measurements (see text). Magnetization versus field at $T=6 \mathrm{~K}(\boldsymbol{\square})$, measured parallel to the substrate plane and scaled to $m^{x y}$, deduced from XMCD (see text and Table I for details). Dotted lines are guides to the eye calculated by scaling the magnetization curve.

approximately 0.3 is significantly smaller than that found in FePc.

\section{DISCUSSION}

The XMCD data shows that the FePc molecules have magnetic $x y$ anisotropy. Due to the textured character of the studied sample, with the molecule planes parallel to the substrate, this microscopic anisotropy is translated into a macroscopic $x y$ anisotropy, which is manifest in the magnetization measurements in parallel and perpendicular directions to the substrate. This result is quite unexpected since $\alpha$-FePc had been reiteratively described as an Ising system, with the moment perpendicular to the molecule plane. ${ }^{19}$ Of course, the FePc film has a different molecular stacking than $\alpha$-FePc, but the molecule anisotropy would be expected to be similar, if not the same. A quite reasonable possibility is that in the bulk the molecular anisotropy axis is $x y$, but since our previous work on $\alpha$-FePc was performed on powder samples, where the anisotropy is averaged in all directions, we were misled to propose the molecule $z$ axis as the magnetic easy axis. Another possibility is that the molecule moment in the $z$ direction is not negligible $\left(m_{z} / m_{x y} \approx 0.5\right)$, so that the averaging procedure we applied to describe the Mössbauer experiments of $\alpha$-FePc are still valid as the $x y$ components would cancel out. At any rate, a very low temperature neutron diffraction experiment would be desirable to determine the magnetic structure of the ferromagnetic bulk $\alpha$-FePc, to sort out its magnetic structure. As to the FePc textured film, to our knowledge, it is beyond the experimental possibility up to date to determine its magnetic ordered structure.

The x-ray absorption spectra of powdered samples has been studied experimentally earlier. In Ref. 26 two configurations were proposed for the electronic ground state: a quintet ${ }^{5} A_{1}$ or a triplet ${ }^{3} E$, but it could not be concluded unambiguously, which was most appropriate. In Ref. 27 the authors conclude by means of multiplet calculations that the 


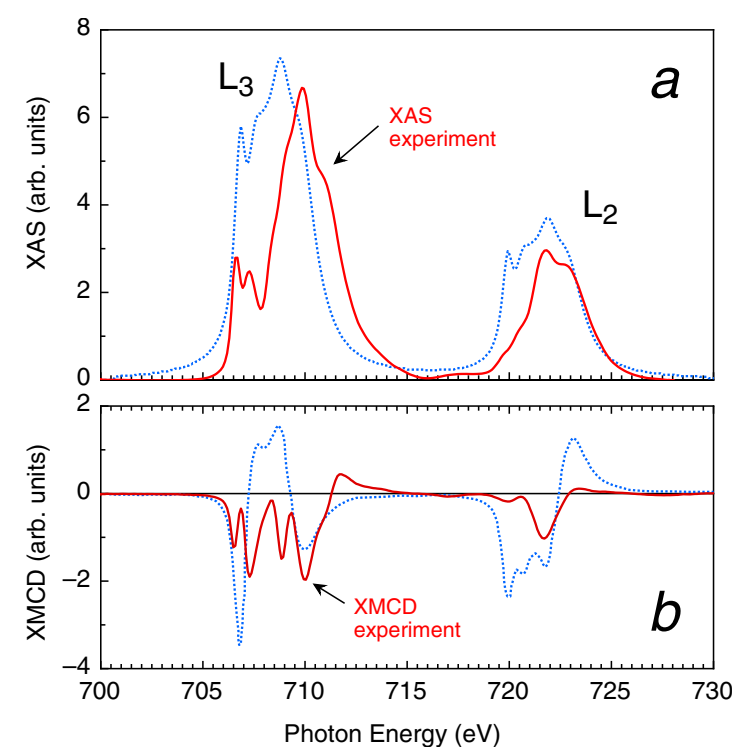

FIG. 8. (Color online) Experimental absorption spectra (red full lines) compared to theoretical predictions from Ref. 17 (dotted blue lines) of $\mathrm{FePc}$ at the $L_{2,3 \mathrm{Fe}}$ edges, for normal incidence, $\gamma=0$. (a) XAS spectra; (b) XMCD spectra. Both panels share the same (arbitrary) units.

ground state configuration is ${ }^{3} E$, under lower symmetry than $D_{4 h}$. They mention that this configuration confirms the ${ }^{3} E$ proposed earlier from Mössbauer experiments, ${ }^{19}$ or from theoretical density functional theory (DFT) calculations on the FePc isolated molecule. ${ }^{17}$ In fact, the empty state configuration given in Ref. 27 propose the existence of holes in $b_{1}, b_{1}$, $b_{2}$, and $e$ states (in $C_{4 v}$ symmetry), while from the present XLPA measurements on textured FePc sample, the proposed configuration has holes in the $b_{1 g}, b_{1 g}, a_{1 g}$, and $e_{g}$ states in $D_{4 h}$ symmetry. Thus, there is a discrepancy whether the $a_{1}$ $\left(a_{1 g}\right)$ or $b_{2}\left(b_{2 g}\right)$ is the level yielding the peak at $707.3 \mathrm{eV}$. The selection rule applied to assign that peak actually excludes the possibility that it is due to a $b_{2}$ state since in that case the peak should have highest intensity in normal incidence $(\vec{E} \| x, y$ plane $)$ and zero or nearly zero for grazing incidence, contrary to our observation. In view of this argument, we propose that the ground state configuration is the triplet ${ }^{3} E_{g}$ type $a$, proposed earlier as deduced from x-ray diffraction ${ }^{18}$ and Mössbauer measurements. ${ }^{19,20}$

The experimental XANES and XMCD spectra are shown in Fig. 8 together with the calculated ones in Ref. 17. For comparison, we note that in that work the energies of the excitations are given with respect to the first, leftmost peak. Instead, in the present work we take as zero energy that of the Fermi level determined as the energy of maximum slope in the low energy tail of $L_{3}$ or $L_{2}$ edges, respectively. Both the experimental and calculated XANES spectra show two lines in the low energy tails of the $L_{2,3}$ edges. The first peak is interpreted in this work as the excitation of $0.3 \mathrm{eV}$ to the hole in the spin-orbit split $e_{g}^{\uparrow}$ level, while it was taken as the reference of zero energy at the $e_{g}^{\uparrow}$ level in Ref. 17 . The second peak corresponds to the $a_{g}^{\uparrow}$ excited level of the minority spin states, which increases its intensity with increasing $\gamma$. The most intense peaks at the right of the $L_{3}$ spectra were ascribed in Ref. 17 from right to left, to $b_{1 g}^{\uparrow}, b_{1 g}^{\downarrow}$, and $e_{g}^{\downarrow}$ states, at $2.99,1.96$, and $1.24 \mathrm{eV}$, respectively. Our experimental spectrum resembles the predicted one, with energies $4.64,3.52$, and $2.57 \mathrm{eV}$, respectively. The two $b_{1 g}$ peak intensities decrease for increasing $\gamma$, as predicted. The peak at $2.57 \mathrm{eV}$ would be assigned to the $e_{g}^{\uparrow}$ state, which stems from the hybridization with the ligand $\pi$ orbital, according to the calculated level scheme. The mentioned difference in reference energy accounts partially to the different results in the excitation energies, but the approximations done in that $a b$ initio calculation are the cause of the larger difference in the high energy excitations.

Another DFT calculation of the electronic structure of FePc ${ }^{28}$ contrasted to ultraviolet photoemission spectroscopy (UPS) experiments, proposes rather a ${ }^{3} B_{2 g}$ or ${ }^{3} A_{1 g}$ ground state electronic configurations, and disregard the ${ }^{3} E_{g}$ ones. However, these configurations do not allow by symmetry the large orbital moment found, since the orbital degeneracy originated at the $e_{g}$ level disappears. This result is in contrast with our results and with the previously discussed DFT calculations. ${ }^{17}$ New calculations of XAS, XLPA, and $\mathrm{XMCD}$, including angle dependence, are necessary to solve unambiguously this question, though it seems that FePc has a ${ }^{3} E_{g}$ ground state configuration is a quite robust result.

The present results showing a largely unquenched orbital moment are in qualitative agreement with the following model. The $\mathrm{Fe}(\mathrm{II})-N$ bond is time-shared between the two pairs of opposite $N$ atoms, giving rise to orbital degeneracy of the $e_{g}\left(d_{x z} d_{y z}\right)$ level. In the spin-split ligand-field energy level scheme for FePc supported by our experiments (Fig. 5), an unoccupied state is available at the $e_{g}\left(d_{x z} d_{y z}\right)$, and a second at the $a_{1 g}\left(d_{3 z^{2}-r^{2}}\right)$ level. The spin-up electron on the $e_{g}$ level may occupy either the $d_{x z}$ or the degenerate $d_{y z}$ orbital component of the doublet. The hole-hole interaction between the $e_{g}\left(d_{x z} d_{y z}\right)$ and the $a_{1 g}\left(d_{3 z^{2}-r^{2}}\right)$ holes and the spin-orbit coupling split the degenerate states into three doublet states, with the ground state being the doublet with $\left\langle L_{z}\right\rangle= \pm \hbar$. The orbital moment of the free $\mathrm{Fe}(\mathrm{II})$ is, therefore, only partially quenched. A second-order perturbation spin-orbit or crystal field perturbation (for example, a lowering of symmetry by a small distortion of the molecule to $D_{2 h}$ ) would split this doublet, therefore yielding to an occupied and an unoccupied $e_{g}$ level with $a_{1 g}$ mixing. The resulting ground state is that of a partially quenched orbital moment. ${ }^{19}$

It is very interesting to note that in recent electron energyloss spectroscopy (EELS) experiments on thin free-standing films of FePc, a low energy excitation from the $a_{1 u}$ ligand states into the $\mathrm{Fe} 3 d\left(e_{g}\right)$ states have been observed. ${ }^{29}$ These are hybridized states with the ligand $e_{g}$ states and a necessary condition for its observation is that the $\mathrm{Fe} 3 d\left(e_{g}\right)$ are not fully occupied. This experimental observation is regarded by those authors as proof of the soundness of our model to explain the large orbital moment found, first proposed in Ref. 19.

The dipolar term has a strong contribution to $m_{S}^{\text {eff }}$ in this case. Since $m_{T}(\gamma)=\hat{T}_{\gamma} \mu_{B} / \hbar$ one obtains $T_{x x}=T_{y y}=-0.037 \hbar$, and therefore, $T_{z z}=0.074 \hbar .{ }^{23}$ These are quite large values, comparable to the calculated value of $\mathrm{Fe}^{2+}$ in a surface site, ${ }^{30}$ and is related to the planar symmetry of the ligand field in FePc. 
With the experimentally determined values of $m_{L}^{x y}, m_{L}^{z}$, and $m_{T}^{z}$ it is possible to derive an estimation of the magnetic anisotropy energy originating from spin-orbit coupling by means of the expression

$$
\Delta E_{S O}=-\frac{G}{H}\left\{\frac{\xi_{3 d}\left(m_{L}^{z}-m_{L}^{x y}\right)}{4 \mu_{B}}+\frac{\xi_{3 d}^{2}\left[\left(\frac{21}{2}\right)\left(\frac{3}{2}\right) m_{T}^{z}+A\right]}{\Delta E_{e x} \mu_{B}}\right\},
$$

where $\mathrm{G} / \mathrm{H} \approx 0.2$ for transition metals, $\xi_{3 d}=0.05 \mathrm{eV}$ for $\mathrm{Fe},{ }^{31-36}$ and $\Delta E_{e x}=1 \mathrm{eV}$ is the energy shift between the majority and minority states. This value is extracted from our spectroscopic results as the energy difference between the $b_{1 g}$ minority and $b_{1 g}$ minority energy levels. The term $\mathrm{A}$ is independent of the spin direction and may be considered as negligible. The first term corresponds to the anisotropy originating from the difference in orbital moment between the perpendicular and parallel directions to the substrate plate (thus of orbital origin), and the second term from the intraatomic dipolar term, describing the anisotropy in the spin density. Substituting, one obtains: $\Delta E_{S O} \approx+6.0 \times 10^{-4}-5.3$ $\times 10^{-4}=7 \times 10^{-5} \mathrm{eV}$. Thus, the orbital term and the dipolar term are of the same order of magnitude and are competing with each other. The sign is positive, thus favoring $x y$ anisotropy. Since the anisotropy field was estimated as $\mu_{0} H_{A}$ $=0.6 \mathrm{~T}$, the anisotropy (volume) constant can be evaluated as $\left|K_{1}\right|=\mu_{0} H_{A} M_{s} / 2$, yielding $K_{1}=-2.2 \times 10^{3} \mathrm{~J} / \mathrm{m}^{3}$, or $\Delta E_{S O}=2.1 \times 10^{-5} \mathrm{eV} / \mathrm{at}$, assuming the specific volume of $\mathrm{Fe}$ atoms as $v=1.48 \times 10^{-28} \mathrm{~m}^{3}$, as in $\alpha$-FePc. The experimental value is of the same order of magnitude as the theoretical estimation from Eq. (7), although somewhat lower. Of course, Eq. (7) is just a rough approximation since the original model is derived for a fully occupied majority band, and a completely empty minority band, which is not our case, but it justifies qualitatively our finding of $x y$ anisotropy in FePc.

\section{CONCLUSION}

The FePc film deposited on a smooth Au substrate, with the molecule planes lying flat on the substrate plane, as demonstrated by x-ray diffraction and XLPA measurements on the $N$ K-edge, has $x y$ anisotropy, as shown by magnetization measurements below the magnetic ordering temperature $T_{C}$ $=4 \mathrm{~K}$. Besides, the XLPA angle-dependent spectra at the $L_{2,3} \mathrm{Fe}$ edges have been indexed in terms of the ${ }^{3} E_{g}$ type $a$ electronic configuration.

From the XMCD measurements at the $L_{2,3}$ Fe edges, the xy anisotropy is also demonstrated and an unusually large orbital moment in FePc molecule is found. This happens, in spite of the low symmetry of the molecule, because of the large orbital degeneracy caused by the time sharing of the two opposite Fe-N bond pairs. Similar behavior may be expected in other very interesting $\mathrm{Fe}^{2+}$ planar molecules, such as in $\alpha$-Fe OETA-Porphyrin, where also a very large hyperfine field $\left(B_{h f}=62.1 \mathrm{~T}\right)$ has been measured. ${ }^{37}$

To obtain a large orbital moment the specific conditions found are: (a) planar symmetry yielding to a level scheme, in increasing energy, $b_{2 g}, e_{g}, a_{1 g}$, and $b_{1 g}$, (b) the electron occupancy of these levels leaves the $e_{g}$ incompletely filled, thereby creating orbital degeneracy, and (c), a second-order perturbation rendering as ground state a singlet with partially quenched, but high, orbital moment. These conditions are fulfilled by $\mathrm{Fe}$ (II) in the FePc molecule. One can view this as a general result, and may help in the search of new molecules with high magnetic anisotropy.

\section{ACKNOWLEDGMENTS}

The financial support of Spanish MAT08/1077 and Aragonese E-34 and E-69 projects, the U.S. NSF DMR-grant under Grant No. 0847552, U.S. Department of Energy and the UCOP program on Carbon-based nanostructures, and Romanian contract 235-IDEI Program are acknowledged. The authors are thankful to J. Ferrer, M. D. Kuz'min, A. Labarta, R. Natoli, J. García, J. Camarero, S. K. Sinha, H. Suhl, and P. Gambardella for fruitful discussions. This work corresponds to ESRF experiment HE2486.

\footnotetext{
*barto@unizar.es

${ }^{1}$ Phthalocyanines, Properties and Applications, edited by C. C. Leznoff and A. B. P. Lever (VCH, New York, 1996), Vol. 1-4.

${ }^{2}$ J. H. van Vleck, The Theory of Electric and Magnetic Susceptibilities (Clarendon Press, Oxford, 1932).

${ }^{3}$ P. Ghigna, A. Campana, A. Lascialfari, A. Caneschi, D. Gatteschi, A. Tagliaferri, and F. Borgatti, Phys. Rev. B 64, 132413 (2001).

${ }^{4}$ R. Moroni, C. Cartier dit Moulin, G. Champion, M.-A. Arrio, P. Sainctavit, M. Verdaguer, and D. Gatteschi, Phys. Rev. B 68, 064407 (2003).

${ }^{5}$ M. Tischer, O. Hjortstam, D. Arvanitis, J. Hunter Dunn, F. May, K. Baberschke, J. Trygg, J. M. Wills, B. Johansson, and O. Eriksson, Phys. Rev. Lett. 75, 1602 (1995).

${ }^{6}$ P. Gambardella, A. Dallmeyer, K. Maiti, M. C. Malagoli, W. Eberhardt, K. Kern, and C. Carbone, Nature (London) 416, 301
}

(2002).

${ }^{7}$ K. W. Edmonds, C. Binns, S. H. Baker, S. C. Thornton, C. Norris, J. B. Goedkoop, M. Finazzi, and N. B. Brookes, Phys. Rev. B 60, 472 (1999).

${ }^{8}$ P. Gambardella et al., Science 300, 1130 (2003).

${ }^{9}$ P. Gambardella, S. S. Dhesi, S. Gardonio, C. Grazioli, P. Ohresser, and C. Carbone, Phys. Rev. Lett. 88, 047202 (2002).

${ }^{10}$ R. Kirsch, M. Prandolini, O. Beutler, M. Gruyters, J. Kapoor, D. Riegel, H. Ebert, and S. Frota-Pessoa, EPL 59, 430 (2002).

${ }^{11}$ M. Evangelisti, J. Bartolomé, L. J. de Jongh, and G. Filoti, Phys. Rev. B 66, 144410 (2002).

${ }^{12}$ S. Yim, S. Heutz, and T. S. Jones, Phys. Rev. B 67, 165308 (2003).

${ }^{13}$ C. W. Miller, A. Sharoni, G. Liu, C. N. Colesniuc, B. Fruhberger, and I. K. Schuller, Phys. Rev. B 72, 104113 (2005).

${ }^{14}$ G. Liu, T. Gredig, and I. K. Schuller, EPL 83, 56001 (2008). 
${ }^{15}$ M. L. M. Rocco, K.-H. Frank, P. Yannoulis, and E.-E. Koch, J. Chem. Phys. 93, 6859 (1990).

${ }^{16}$ J. Stöhr and H. Siegmann, Magnetism: From Fundamentals to Nanoscale Dynamics (Springer-Verlag, Berlin, Heidelberg, 2006).

${ }^{17}$ M. D. Kuz'min, R. Hayn, and V. Oison, Phys. Rev. B 79, 024413 (2009).

${ }^{18}$ P. Coppens, L. Li, and N. J. Zhu, J. Am. Chem. Soc. 105, 6173 (1983).

${ }^{19}$ G. Filoti, M. D. Kuz'min, and J. Bartolomé, Phys. Rev. B 74, 134420 (2006).

${ }^{20}$ B. Dale, R. Williams, P. Edwards, and C. Johnson, J. Chem. Phys. 49, 3445 (1968).

${ }^{21}$ B. T. Thole, P. Carra, F. Sette, and G. van der Laan, Phys. Rev. Lett. 68, 1943 (1992).

${ }^{22}$ P. Carra, B. T. Thole, M. Altarelli, and X. Wang, Phys. Rev. Lett. 70, 694 (1993).

${ }^{23}$ J. Stöhr and H. König, Phys. Rev. Lett. 75, 3748 (1995).

${ }^{24}$ P. Gambardella et al., Nat. Mater. 8, 189 (2009).

${ }^{25}$ L. Paolasini et al., Phys. Rev. Lett. 82, 4719 (1999).

${ }^{26}$ B. T. Thole, G. van der Laan, and P. H. Butler, Chem. Phys. Lett. 149, 295 (1988).

${ }^{27}$ P. Miedema, S. Stepanow, P. Gambardella, and F. M. F. de Groot, J. Phys.: Conf. Ser. 190, 012143 (2009).
${ }^{28}$ N. Marom and L. Kronik, Appl. Phys. A: Mater. Sci. Process. 95, 165 (2009).

${ }^{29}$ A. König, F. Roth, R. Kraus, and M. Knupfer, J. Chem. Phys. 130, 214503 (2009).

${ }^{30}$ J. Crocombette, B. Thole, and F. Jollet, J. Phys.: Condens. Matter 8, 4095 (1996).

${ }^{31}$ G. van der Laan, J. Phys.: Condens. Matter 10, 3239 (1998).

${ }^{32}$ D. Weller, J. Stöhr, R. Nakajima, A. Carl, M. G. Samant, C. Chappert, R. Mégy, P. Beauvillain, P. Veillet, and G. A. Held, Phys. Rev. Lett. 75, 3752 (1995).

${ }^{33}$ K. Mamiya, T. Koide, Y. Ishida, Y. Osafune, A. Fujimori, Y. Suzuki, T. Katayama, and S. Yuasa, Radiat. Phys. Chem. 75, 1872 (2006).

${ }^{34}$ In Refs. 31 and 33, eqn. 7 is given for the sign criteria of the rule sum as $m_{S}^{\text {eff }}=m_{S}+7 m_{T}$. We are using, instead, $m_{S}^{\text {eff }}=m_{S}-7 m_{T}$ following Stöhr (Ref. 16) and Weller (Ref. 32), and therefore, there is a change of sign in the second term in eqn. 7 with respect to that given by van der Laan (Ref. 31) and Mamiya (Ref. 33).

${ }^{35}$ D. S. Wang, R. Wu, and A. J. Freeman, Phys. Rev. B 47, 14932 (1993).

${ }^{36}$ P. Bruno, Phys. Rev. B 39, 865 (1989).

${ }^{37}$ W. M. Reiff, C. M. Frommen, G. T. Yee, and S. P. Sellers, Inorg. Chem. 39, 2076 (2000). 